\title{
The expression and impact of antifungal grooming in ants
}

\author{
A. REBER, J. PURCELL, S. D. BUECHEL ${ }^{1}$, P. BURI ${ }^{2}$ \& M. CHAPUISAT \\ Department of Ecology and Evolution, University of Lausanne, Lausanne, Switzerland
}

Keywords:

antiparasite behaviour; entomopathogenic fungus; grooming; social immunity; social insects.

\begin{abstract}
Parasites can cause extensive damage to animal societies in which many related individuals frequently interact. In response, social animals have evolved diverse individual and collective defences. Here, we measured the expression and efficiency of self-grooming and allo-grooming when workers of the ant Formica selysi were contaminated with spores of the fungal entomopathogen Metarhizium anisopliae. The amount of self-grooming increased in the presence of fungal spores, which shows that the ants are able to detect the risk of infection. In contrast, the amount of allo-grooming did not depend on fungal contamination. Workers groomed all nestmate workers that were re-introduced into their groups. The amount of allo-grooming towards noncontaminated individuals was higher when the group had been previously exposed to the pathogen. Allo-grooming decreased the number of fungal spores on the surface of contaminated workers, but did not prevent infection in the conditions tested (high dose of spores and late allo-grooming). The rate of disease transmission to groomers and other nestmates was extremely low. The systematic allo-grooming of all individuals returning to the colony, be they contaminated or not, is probably a simple but robust prophylactic defence preventing the spread of fungal diseases in insect societies.
\end{abstract}

\section{Introduction}

One of the major unresolved questions in evolutionary biology is whether diseases prevent or promote social behaviour. On the one hand, diseases might constrain social evolution (Hamilton, 1987). Social life is associated with a high frequency of contacts among individuals, which is likely to increase the risk of disease transmission (Fefferman et al., 2007; Naug, 2008). Moreover, insect societies usually contain related individuals that remain together in long-lasting nests, providing ideal conditions for the spread of epidemics once the disease has broken into the colony (Hamilton, 1987; Schmid-Hempel, 1998).

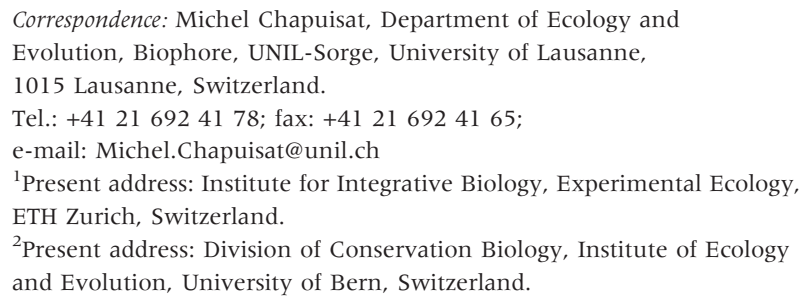

On the other hand, over evolutionary time, social organisms may be able to reduce the impact of disease by evolving collective defences that limit the spread of infection (Cremer et al., 2007; Wilson-Rich et al., 2009; Chapuisat, 2010). In insect societies, many of these defences are behavioural and social and aim at improving the nest hygiene. For example, workers of most species exhibit elaborate waste management behaviours and carry corpses out of their nests (Hart \& Ratnieks, 2002; Cremer et al., 2007; Wilson-Rich et al., 2009).

Both solitary and social organisms can groom themselves (self-grooming), but social organisms have the additional advantage of being able to groom each other (allo-grooming). Allo-grooming is an important line of defence in many social animals, because it permits the efficient mechanical removal of parasites such as mites, fleas, nematodes, fungal hyphae or fungal spores from areas that are not easily accessed during self-grooming (Schmid-Hempel, 1998). Allo-grooming is common in mammals, particularly primates, ungulates and some rodents, where it is generally considered to be a form of reciprocity (Mooring et al., 2004; Clutton-Brock, 
2009). Allo-grooming is also very widespread in the social insects, where it has been documented in many species of ants, wasps, bees and termites (Rosengaus et al., 1998b; Schmid-Hempel, 1998; Hughes \& Boomsma, 2004; Walker \& Hughes, 2009).

In addition to the removal of parasites, allo-grooming has important social functions. In mammals, allo-grooming plays a major role in social bonding (Clutton-Brock, 2009; Dunbar, 2010). In the social insects, allo-grooming and trophallaxis (oral exchange of liquid food) result in a transfer of chemical cues among individuals, and this homogenization of the colony odour is important for accurate nestmate discrimination (Boulay et al., 2004). Hence, the social and hygienic roles of allo-grooming are often confounded.

A better understanding of the hygienic function of allo-grooming requires the estimation of its benefits and costs when a group is exposed to parasites. The potential benefits of allo-grooming stem from the removal of parasites, which may increase the survival of the individual being groomed and/or reduce the withingroup transmission of parasites if groomers have means to kill the parasites or inactivate infectious particles (Cremer et al., 2007). The costs of allo-grooming include the time and energy spent in grooming, as well as the possibility that the contact with a parasitized individual increases the risk of parasite transmission to the groomers (Rosengaus \& Traniello, 1997; Schmid-Hempel, 1998; Cremer et al., 2007).

The expression of allo-grooming is likely to depend on the presence and location of the parasitic threat. Allogrooming is generally considered to be a conditional response to the presence of dangerous parasites. For example, the frequency of allo-grooming increased in groups of termites and ants that had been experimentally contaminated with Metarhizium anisopliae fungal spores (Rosengaus et al., 1998b; Hughes \& Boomsma, 2004; Walker \& Hughes, 2009). However, allo-grooming might also be a constitutive defence that is systematically applied to individuals that are particularly likely to carry an infection, independent of the actual presence of parasites. Leafcutter ants engage in proactive self-grooming before entering their nests, particularly when the nest chambers contain sensitive fungal crops or brood (Morelos Juárez et al., 2010). Similarly, the systematic allo-grooming of individuals returning to their nest after a foraging trip might be a way to remove undetected microbes before they could be transmitted to nestmates.

The way in which a group responds to parasites may also depend on characteristics of both the parasites and the group. In particular, the amount and efficiency of self- or allo-grooming might vary according to infection dose and infection stage, as removing spores may become increasingly difficult at high doses or when the fungus has had time to attach to the cuticle (Hajek \& St. Leger, 1994). Allo-grooming efficiency may also vary with group size. For example, larger groups might be able to allocate more individuals to allo-grooming and thus remove spores more efficiently than smaller groups (Schmid-Hempel, 1998). On the other hand, smaller groups might have more to lose if one of their members dies or is excluded and might thus put relatively more effort into allo-grooming.

In this study, we examined the expression and impact of antifungal grooming in the ant Formica selysi. We exposed groups of workers to spores of the fungus $M$. anisopliae. This generalist entomopathogen is common in the habitat of F. selysi (A. Reber \& M. Chapuisat, unpublished data). In a series of experiments, we examined which factors affected the behavioural response of ants and evaluated the impact of allo-grooming on groomed individuals and grooming individuals. First, we recorded the amount of self-grooming and allo-grooming in groups of workers exposed to various doses of fungal spores deposited in their nest chamber. Second, we examined the allo-grooming rate and its efficiency at removing fungal spores when one contaminated individual was re-introduced into its group, which mimics the situation where a forager returns to the nest. We tested the effect of contamination, time elapsed since contamination and group size. Third, we assessed whether allo-grooming and social contacts increased the survival of ants that had been exposed to a high dose of spores $24 \mathrm{~h}$ before being groomed. Finally, we evaluated the risk of disease transmission from contaminated ants to groomers or other group members. Taken together, these experiments shed light on the modalities and impact of grooming as a means of defence against fungal infection in insect societies.

\section{Materials and methods}

\section{General procedure}

We studied grooming in the ant F. selysi. This species builds permanent nests in the soil. Workers forage above ground for insect prey and aphid honeydew, which they collect and bring back to the nest (Keller \& Zettel, 2001). Hence, like many other ants, F. selysi workers are likely to have frequent contact with spores of soil-dwelling entomopathogenic fungi. We sampled $F$. selysi workers from a population located along the river Rhône between Sierre and Susten in the central Valais, Switzerland. In summer 2007, we collected 300 workers from each of 43 singlequeen colonies (Chapuisat et al., 2004; Reber et al., 2008). The workers were kept in plastic boxes $(13.5 \times 15 \times 5 \mathrm{~cm})$ lined with fluon, at $25^{\circ} \mathrm{C}$ and under a 12-h day/night cycle. The ants had ad libitum access to water and a protein-rich jelly food.

We challenged the ants with $M$. anisopliae var. anisopliae, an entomopathogenic fungus that is common in the Swiss lowlands (Keller et al., 2003). We used a fungus strain isolated from the soil of our study site and causing high mortality to F. selysi workers (Reber et al., 2008; 
A. Reber \& M. Chapuisat, unpublished data). The fungus was cycled four times in F. selysi workers and cultured on a nutritive medium (malt extract agar; BD, Franklin Lake, NJ, USA) at $25^{\circ} \mathrm{C}$ for $5-7$ days to obtain asexual spores (conidia). The spores were harvested into sterile $0.05 \%$ Tween 20 buffer (the nontoxic, highly diluted polysorbate surfactant Tween 20 was used to keep spores in suspension). The concentration of spores was measured with a haemocytometer (Neubauer improved counting chamber). Water and $0.05 \%$ Tween 20 buffer were used in controls.

Experimental groups of ants were formed $24 \mathrm{~h}$ before the beginning of the experiments and were provided with ad libitum food and water. We removed water tubes and food during behavioural observations. The observers were blind with respect to the treatments, because a third person applied the treatments and anonymized the labels at the beginning of the experiments.

Because the effect of allo-grooming on spore removal is likely to depend on the stage of infection and in particular on the degree of attachment of spores, we measured spore removal efficiency when allo-grooming occurred 1 and $24 \mathrm{~h}$ after contamination, respectively. This timing was adjusted to the development of the infection in insects: $M$. anisopliae conidia germinate and develop an attachment structure (appressorium) between 12 and 24 h (Hajek \& St. Leger, 1994; Moino et al., 2002).

To assess whether the mortality occurring during the experiments was caused by $M$. anisopliae, all corpses were removed from experimental boxes, surface-sterilized (Lacey, 1997) and placed in tubes with wet cotton wool. These tubes were kept at $25{ }^{\circ} \mathrm{C}$ and corpses were visually inspected to detect the presence of green stacks of spores that diagnose $M$. anisopliae infection.

\section{Experiment 1: Grooming after a fungal contamination of the group}

We assessed the amount of self-grooming and allogrooming when group members were jointly exposed to fungal spores. We established 95 experimental groups of 11 workers in Petri dishes $(9 \mathrm{~cm}$ diameter) lined with fluon. Workers from 19 field colonies were split into five experimental groups per colony, each group being randomly assigned to one of the five following treatments: sterile water, $0.05 \%$ Tween 20 buffer, low, medium and high concentrations of Metarhizium spores $\left(10^{4}, 10^{6}\right.$ and $10^{8}$ conidia $\mathrm{mL}^{-1}$ of $0.05 \%$ Tween 20 buffer, respectively). Two hundred microlitres of solution was deposited with a micropipette on a filter paper placed in the centre of the Petri dish.

The behaviour of ants was observed for $30 \mathrm{~s}$ at 10, 30 and 70 min after the treatment. We recorded the occurrence of self-grooming (one single ant cleaning herself using her mandibles or legs) and allo-grooming (one ant being cleaned by one or more other ants). We evaluated the overall effect of treatments on the occurrence of selfand allo-grooming with Kruskal-Wallis tests. When the overall effect of treatments was significant, we further investigated the differences between pairs of treatments with Mann-Whitney tests for independent data.

\section{Experiment 2: Allo-grooming of contaminated nestmates re-introduced into their group}

We observed the behaviour of ants towards contaminated group members that were returned to the group after a period of isolation. We established 40 experimental groups of 30 workers in plastic boxes $(13.5 \times 15 \times 5 \mathrm{~cm})$ lined with fluon. The workers originated from 10 field colonies. In two experimental groups (=replicates) per colony, a worker was re-introduced after having been isolated for $\mathrm{l} \mathrm{h}$ (before conidia had adhered to the cuticle), whereas in the two other experimental groups, a worker was re-introduced after $24 \mathrm{~h}$ (after conidia had germinated and adhered to the cuticle). Two workers were removed from each experimental group. For half of the groups (ten groups from the 1 -h treatment and ten groups from the 24-h treatment), these two workers were contaminated with fungal spores by depositing on their thorax $1 \mu \mathrm{L}$ of $M$. anisopliae at a concentration of $10^{7}$ conidia $\mathrm{mL}^{-1}$ (we used a high dose to ensure that enough conidia would stay on the cuticle, as many are lost during the application process, see results of experiment 3). For the other 20 groups, these two workers were uncontaminated controls that received $1 \mu \mathrm{L}$ of sterile $0.05 \%$ Tween 20 buffer. Each ant removed from the group was isolated in a small Petri dish $(3.5 \mathrm{~cm}$ diameter) with water and food. One ant was then returned to its group (after 1 or $24 \mathrm{~h}$ since contamination), whereas the other one was kept in isolation as a control that could self-groom but was prevented from receiving allo-grooming.

We observed the behaviour of group members for $10 \mathrm{~min}$ after the re-introduction of the contaminated or control worker. We recorded the number of individuals that allo-groomed the re-introduced ant (=number of allo-groomers) and the total time during which the re-introduced ant was allo-groomed by one or more ants (=duration of allo-grooming).

Forty-eight hours after the first round of behavioural observations, the experiment was repeated, but the treatments were reversed, so that groups that had first received a contaminated worker received a control one, and vice versa. Hence, each experimental group received one contaminated and one control ant, in randomized order. Two groups had to be removed from the analysis because we lost sight of the re-introduced ant during the observation.

The effect of the fungal treatment, round of observation (first or second ant being re-introduced) and time since contamination on the number of allo-groomers and duration of allo-grooming were tested with three-way Anova. We used a mixed model with three fixed factors, 
the fungal treatment (contaminated or control), round of observation and time elapsed between contamination and re-introduction ( 1 or $24 \mathrm{~h}$ ), and a random factor, the field colony from which the workers originated. Nonsignificant terms were sequentially removed, using likelihood ratio tests. In addition, we used a Pearson's correlation test to examine whether the number of allo-groomers was correlated with the duration of allo-grooming.

We performed two additional control experiments. First, we assessed whether the $0.05 \%$ Tween 20 buffer elicited grooming. Second, we evaluated whether the amount of self-grooming before and after re-introduction differed between control and contaminated ants (see Appendix S1).

\section{Experiment 3: Effect of allo-grooming on spore removal}

To estimate the impact of allo-grooming on spore removal, we compared the number of spores remaining on contaminated workers that had been re-introduced in their groups or kept in isolation (Hughes et al., 2002). Immediately after the 10-min period of grooming and observation, groomed and isolated ants were placed individually in microcentrifuge tubes filled with $500 \mu \mathrm{L}$ of $0.05 \%$ Tween 20 . The tubes were vortexed for $1 \mathrm{~min}$ to re-suspend all remaining spores. The Tween solution was completely evaporated by placing the tubes in a speedvac for $6 \mathrm{~h}$ and the spores were re-suspended in $30 \mu \mathrm{L}$ of $0.05 \%$ Tween 20 . For each sample, we took three independent measures of the concentration of conidia with a Neubauer haemocytometer and calculated the total number of spores remaining on the surface of the worker from the average of these three measures.

The effects of allo-grooming and time since contamination on the number of spores remaining on the surface of the contaminated ant were tested with two-way ANova. The number of spores was sin $(x+360)$ transformed to ensure normality and homoscedasticity. We used a mixed model with two fixed factors, the social treatment (allo-groomed or kept in isolation) and time elapsed between contamination and re-introduction (1 or $24 \mathrm{~h}$ ), as well as a random factor, the field colony from which the workers originated. In addition, we used a Kendall's rank correlation test to examine whether the number of spores remaining on contaminated ants was correlated with the duration of allo-grooming.

To detect whether the fungal disease had been transmitted from contaminated ants to groomers or other group members, we monitored the mortality in the experimental groups until 7 days after the end of the second re-introduction and checked whether the corpses produced Metarhizium spores.

\section{Experiment 4: Effect of group size on spore removal}

We also examined whether spore removal by allogrooming increased with group size. For this, we re-introduced contaminated ants in small (three workers) and large (12 workers) groups, respectively. We used workers originating from 29 field colonies to form 29 experimental groups of five workers and 29 experimental groups of 14 workers. The workers were marked with spots of paint to distinguish contaminated and control ants from other group members. The groups were kept in Petri dishes (9 $\mathrm{cm}$ diameter) lined with fluon. Two ants were removed from each experimental group. We contaminated these two ants by depositing on their thorax $1 \mu \mathrm{L}$ of $M$. anisopliae at a concentration of $10^{8}$ conidia $\mathrm{mL}^{-1}$ (only a small proportion of the spores are still on the ant cuticle following the $24 \mathrm{~h}$ in isolation, see Results). We kept each contaminated ant isolated for $24 \mathrm{~h}$ in a small Petri dish $(3.5 \mathrm{~cm}$ diameter) with water and food. One of these contaminated workers was then returned to its group for $1 \mathrm{~h}$, where it was allo-groomed. The other contaminated worker was kept in isolation.

Immediately after this $1-h$ period of grooming or isolation, we measured the number of spores remaining on the body of contaminated workers as described earlier. We used a two-way Anova to test the effect of social treatment (allo-groomed or kept in isolation) and group size on the number of spores remaining on workers. The number of spores was log-transformed to ensure normality and homoscedasticity. As described previously, we also recorded mortality and corpse sporulation to detect whether there had been any transmission of the fungal disease.

\section{Experiment 5: Effect of allo-grooming on the survival of contaminated ants}

To examine the impact of allo-grooming and social contacts on the survival of contaminated ants, we compared the survival of ants that had been kept in isolation, had social contacts but no allo-grooming, or were allo-groomed for $2.5 \mathrm{~h}$ beginning $24 \mathrm{~h}$ after exposure to a high dose of spores. We formed 132 experimental groups of 16 workers. The workers originated from 33 field colonies. Two experimental groups (=replicates) per colony received contaminated ants, whereas the two other experimental groups received control ants. The workers were marked with spots of paint to distinguish contaminated or control ants from other group members. The groups were kept in Petri dishes $(9 \mathrm{~cm}$ diameter) lined with fluon.

Three focal ants were removed from each experimental group. For 66 groups, these three workers were contaminated with fungus by depositing on their thorax $1 \mu \mathrm{L}$ of M. anisopliae at a concentration of $10^{8}$ conidia $\mathrm{mL}^{-1}$, as in experiment 4 . For the other 66 groups, the three workers received $1 \mu \mathrm{L}$ of sterile $0.05 \%$ Tween 20 control buffer. Each contaminated or control worker was kept isolated for $24 \mathrm{~h}$ in a small Petri dish $(3.5 \mathrm{~cm}$ diameter) with water and food. Two workers were then returned to their group. One of the two could be allo-groomed by the 
other group members. The other was placed in a small Petri dish covered by mesh, allowing social contacts such as antennal touching or trophallaxis, but preventing allogrooming. The third worker was kept in isolation as a control that could self-groom but was deprived of allogrooming and social contacts. After $2.5 \mathrm{~h}$, the two re-introduced ants were removed from their experimental groups and kept in isolation. Over the next 10 days, we monitored the mortality of the focal ants and checked whether their corpses produced Metarhizium spores.

We compared the survival of treated and control workers in a parametric survival analysis, using the function survreg of the program R (R Development Core Team, 2006). We built a model with two fixed factors, the fungal treatment (contaminated or control) and social treatment (isolated, having contacts through mesh, or allo-groomed). We included the experimental group nested in the colony of origin as a random factor using the frailty argument for the survreg function (Therneu et al., 2003). The interaction between fixed factors was tested using a likelihood chi-square ratio test. We used a Weibull distribution, which produced the minimum error deviance. All statistical analyses were performed in $\mathrm{R}$ version 2.8.1 (R Development Core Team, 2006).

\section{Results}

\section{Experiment 1: Grooming after a fungal contamination of the group}

Overall, the amount of self-grooming rose significantly when groups of ants were jointly exposed to increasing doses of Metarhizium spores placed on filter paper in the group (Fig. la; $H_{4,90}=11.9, P=0.02$ ). The amount of self-grooming was significantly higher in the presence of high dose of fungal spores than in the presence of Tween control $\left(U=95.5, n_{1}=n_{2}=19, P=0.01\right)$, water control $\left(U=275, n_{1}=n_{2}=19, P=0.005\right)$ or medium dose of spores $\left(U=251, n_{1}=n_{2}=19, P=0.01\right.$; Fig. la), even after a sequential Bonferroni-adjustment of the significance threshold. In contrast, there was no significant effect of treatment on the amount of allo-grooming in this experiment (Fig. $1 \mathrm{~b} ; H_{4,90}=3.6, P=0.5$ ).

\section{Experiment 2: Allo-grooming of contaminated nestmates re-introduced into their group}

When an ant was returned to its group after a short $(1 \mathrm{~h})$ or long $(24 \mathrm{~h})$ period of isolation, it was groomed intensively by an average of five ants and for more than one-third of the total observation time (Fig. 2). All re-introduced ants were groomed, independently of whether they had been contaminated by fungus or not. Indeed, exposing the ant to fungal spores before re-introduction had no significant effect on the number of groomers (Fig. 2a; $F_{1,65}=0.02, P=0.9$ ) nor on the duration of allo-grooming (Fig. $2 b ; F_{1,65}=0.1, P=0.7$ ).
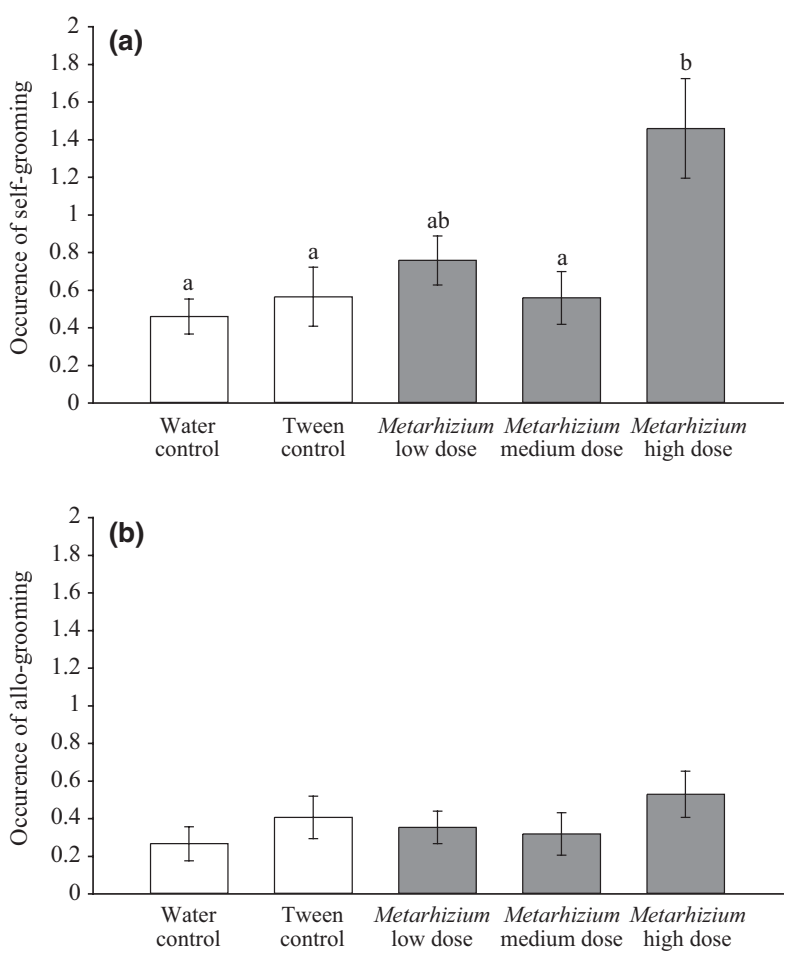

Fig. 1 Expression of (a) self-grooming and (b) allo-grooming when group members were jointly exposed to Metarhizium anisopliae spores. The groups were exposed to water, Tween buffer, low, medium or high dose of Metarhizium deposited on a filter paper, respectively. Mean $( \pm S E)$ occurrence of (a) self-grooming and (b) allo-grooming in groups of 11 individuals, averaged over three observation scans of $30 \mathrm{~s}$ per group. Different letters indicate treatments that differed significantly from one another.

The time elapsed between contamination and re-introduction had no significant effect on the number of allo-groomers (Fig. 2a; $F_{1,65}=0.94, P=0.3$ ) but had a marginally significant effect on allo-grooming duration (Fig. 2b; $F_{1,65}=3.92, P=0.05$ ), which was significantly shorter for control ants re-introduced after $24 \mathrm{~h}$ $\left(F_{1,27}=5.6, \quad P=0.02\right)$. The number of allo-groomers and allo-grooming duration were positively correlated $(r=0.7$, d.f. $=76, P<0.0001)$.

The first and second ants re-introduced were groomed by a similar number of ants (Fig. $3 a ; F_{1,65}=0.9, P=0.3$ ), but overall the second ants being re-introduced were allogroomed for a significantly longer time than the first ones (Fig. 3b; $F_{1,62}=6.9, P=0.01$ ). There was a significant interaction between the round of observation (first or second ant being re-introduced) and the fungal treatment on the duration of allo-grooming (Fig. $3 b ; F_{1,62}=4.1$, $P=0.05)$. The control ants were groomed for a significantly longer time when they were re-introduced following the previous re-introduction of a contaminated ant than when they were the first ones to be re-introduced (Tween control, first vs. second re-introduction: $t_{38}=3.1$, 

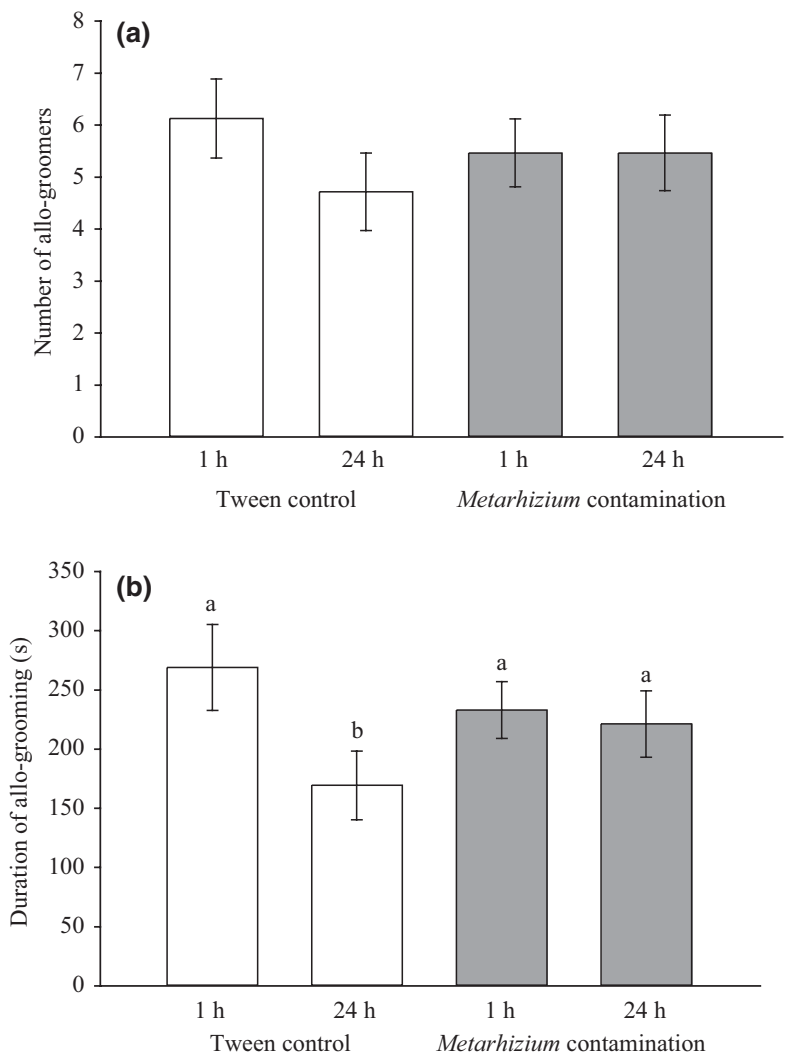

Fig. 2 Expression of allo-grooming when a nestmate contaminated with Metarhizium anisopliae spores was re-introduced in its group. The recipient group had 28 ants and allo-grooming was measured over a period of 10 min. (a) Mean $( \pm \mathrm{SE})$ number of ants allo-grooming the re-introduced ant. (b) Mean $( \pm$ SE) duration of allo-grooming. The expression of allo-grooming is given in function of the contamination of the re-introduced ants (control or Metarhizium-contaminated) and time elapsed between contamination and re-introduction ( 1 or $24 \mathrm{~h}$ ). Different letters indicate treatments that differed significantly from one another.

$P=0.002)$. In contrast, the contaminated ants were not groomed for longer when they were re-introduced after a control ant than when they were the first ones to be re-introduced (Metarhizium-contaminated, first vs. second re-introduction: $\left.t_{38}=0.4 P=0.7\right)$. This suggests that the reaction towards control and contaminated ants differs in subtle ways and that the grooming of noncontaminated ants increases when the group had been previously exposed to the pathogen.

Overall, ants that were treated with $0.05 \%$ Tween 20 buffer, with or without Metarhizium spores, were groomed by about five nestmates for $4 \mathrm{~min}$, on average, whereas ants that had received a drop of sterile water or the dry treatment were allo-groomed by one or two nestmates for about $1 \mathrm{~min}$, on average (Figs 2 and S1). Hence, the application of Tween 20 buffer, with or without spores, elicited a significantly longer and more vigorous allo-grooming interaction than the dry or water
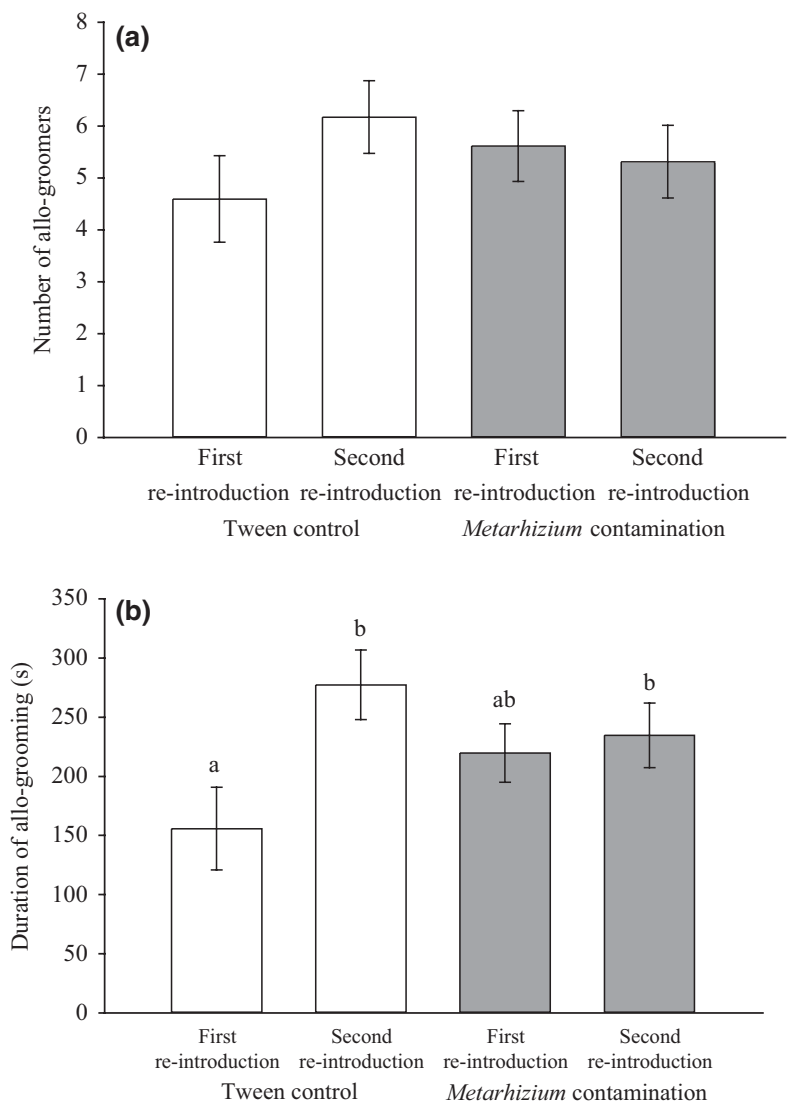

Fig. 3 Expression of allo-grooming towards the first and second nestmate re-introduced in their group (the two ants were re-introduced with an interval of $48 \mathrm{~h}$ ). For each group, the first and second nestmates re-introduced in the group had alternative treatments (control or contaminated, respectively). The recipient group had 28 ants and allo-grooming was measured over a period of $10 \mathrm{~min}$. (a) Mean $( \pm \mathrm{SE})$ number of ants allo-grooming the re-introduced ant. (b) Mean $( \pm \mathrm{SE})$ duration of allo-grooming. The expression of allo-grooming is given in function of the contamination of the re-introduced ants (control or Metarhiziumcontaminated). Different letters indicate treatments that differed significantly from one another.

only treatments (Fig. S1; grooming duration: $F_{5,49}=$ 5.17, $P=0.0007$; number of groomers: $F_{5,49}=5.62$, $P=0.0004)$. However, the proportion of time that nestmates spent allo-grooming re-introduced ants that had only been exposed to the water or dry treatments was significantly higher than the background level of allo-grooming among untreated ants, even when the re-introduced ants had been separated for only $\mathrm{l} \mathrm{h}$ (Fig. S1; background level of allo-grooming was zero for all ten groups observed; Bonferroni-corrected proportion tests $(\alpha=0.025)$ : water treatment vs. background $\chi^{2}=601.8$, d.f. $=1, \quad P<0.0001$; dry treatment vs. background $\chi^{2}=620.6$, d.f. $\left.=1, P<0.0001\right)$. This suggests that the ants systematically allo-groom all ants returning to the 

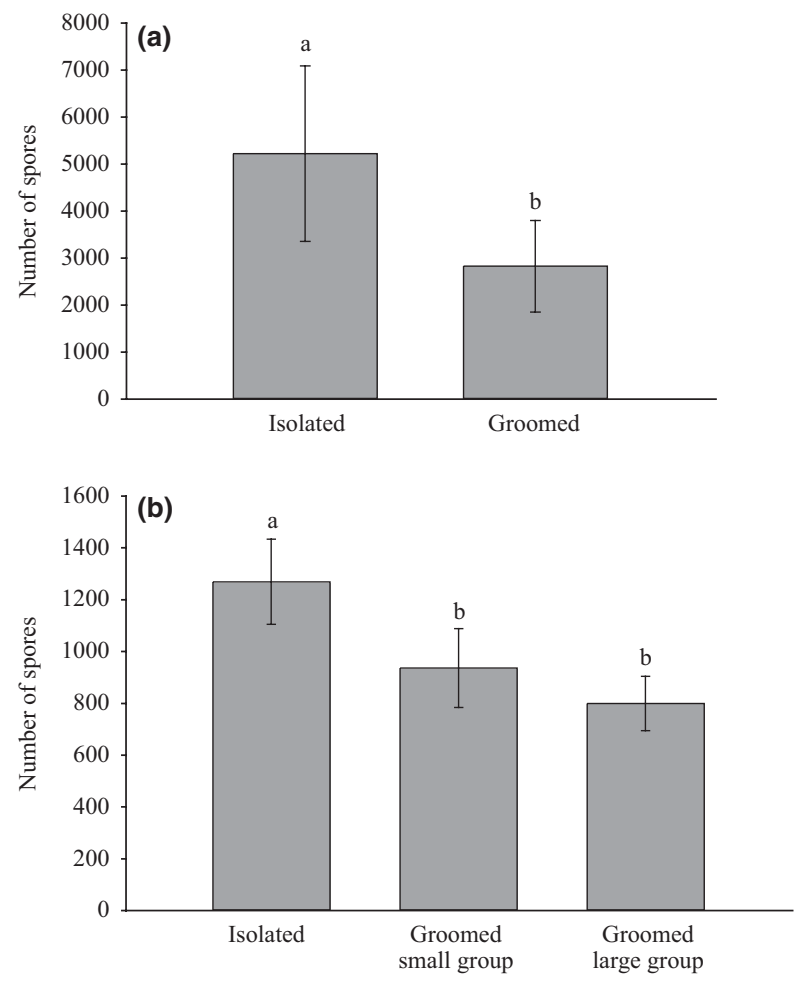

Fig. 4 Effect of allo-grooming and group size on spore removal. (a) Mean $( \pm \mathrm{SE})$ number of spores remaining on the body of Metarhizium-contaminated ants that were kept in isolation or re-introduced in their group (28 ants) for 10 min. (b) Mean $( \pm \mathrm{SE})$ number of spores remaining on the body of Metarhizium-contaminated ants that were kept in isolation, re-introduced in small groups (three ants), or re-introduced in large groups (12 ants) for $1 \mathrm{~h}$. Different letters indicate treatments that differed significantly from one another.

group, independent of the treatment. In the re-introduction experiments, the amount of self-grooming did not vary significantly across treatments, neither when the individuals were observed in isolation (Fig. S1; $\left.F_{5,79}=0.77, P=0.57\right)$ nor when they were observed after returning to their group $\left(F_{5,31}=1.36, P=0.27\right)$.

\section{Experiment 3: Effect of allo-grooming on spore removal}

Contaminated ants re-introduced into their experimental group, where they could be allo-groomed by 28 other ants for up to $10 \mathrm{~min}$, had significantly fewer fungal spores than ants kept in isolation, which could only selfgroom (Fig. $4 a ; F_{1,65}=6.44, P=0.01$ ). Hence, this short period of allo-grooming resulted in a $46 \%$ decrease in the number of spores remaining on the body of contaminated ants, relative to ants that could only self-groom. The number of spores remaining on the ants was negatively correlated with the duration of allo-grooming $\left(t_{38}=-0.2, P=0.05\right)$.
The time elapsed between contamination and re-introduction had no significant effect on the number of spores remaining on the ants $\left(F_{1,65}=0.2, P=0.6\right)$. Moreover, the efficiency of allo-grooming was similar after a short and long period of isolation, as there was no interaction between the social treatment (allogroomed or kept in isolation) and the time elapsed between contamination and re-introduction $\left(F_{1,65}=1.26\right.$, $P=0.2)$.

There was very limited transmission of disease from experimentally contaminated individuals to groomers or other group members. In total, 137 of the 1120 group members that potentially had been in contact with one contaminated nestmate died within 1 week of the second re-introduction, but only one of these 137 corpses produced Metarhizium spores.

\section{Experiment 4: Effect of group size on spore removal}

There were significantly fewer fungal spores on contaminated ants that had been re-introduced in groups of three or twelve ants for $1 \mathrm{~h}$ than on contaminated ants that were kept in isolation (Fig. 4b; $F_{1,116}=4.42$, $P=0.04)$. However, group size had no significant effect on the number of spores remaining after grooming (Fig. 4b; $F_{1,116}=0.1, P=0.7$ ), and there was no significant interaction between social treatment (allo-groomed or kept in isolation) and group size (Fig. $4 \mathrm{~b} ; F_{1,116}=$ $0.0004, P=0.9)$.

We detected no disease transmission from the contaminated ant to other group members. Only 16 of the 435 group members that potentially had been in contact with one contaminated nestmate died during the following week, and none of these 16 corpses produced Metarhizium spores.

\section{Experiment 5: Effect of allo-grooming on the survival of contaminated ants}

The ants experimentally contaminated with Metarhizium spores suffered from a significantly higher mortality than control ants (Fig. 5; mortality after 10 days was 59\% and $5 \%$ for contaminated and control ants, respectively; $\chi^{2}=166.5$, d.f. $\left.=1, P<0.0001\right)$. The elevated mortality in contaminated ants was almost entirely caused by Metarhizium infection, as $90 \%$ of their corpses produced Metarhizium spores. In contrast, none of the corpses from control ants produced Metarhizium spores.

Allo-grooming did not protect the contaminated ants from the Metarhizium infection, at least under the limited conditions tested. Indeed, the survival of contaminated ants did not differ significantly when they could be allogroomed, had social contact through mesh that prevented allo-grooming or were kept in isolation (Fig. 5; $\chi^{2}=0.4$, d.f. $=2, \quad P=0.8$ ). Moreover, there was no interaction between the fungal treatment (contaminated or control) and social treatment (allo-groomed, having 


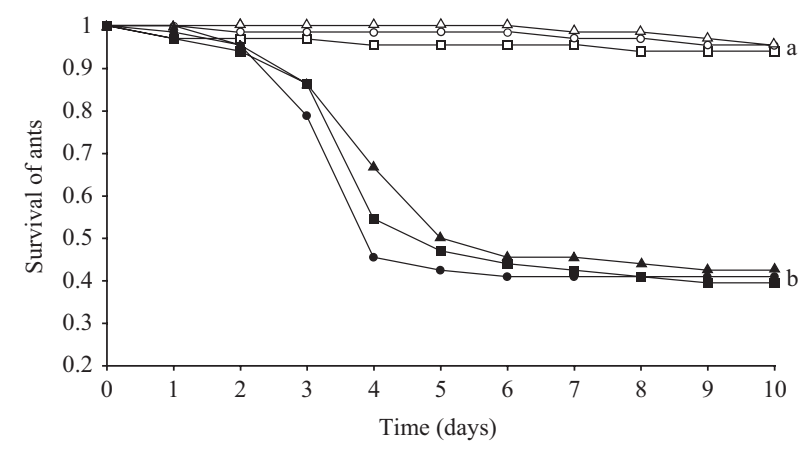

Fig. 5 Effect of allo-grooming and social contacts on the survival of ants exposed to Metarhizium. Control ants (exposed to Tween buffer, open symbols) or Metarhizium-contaminated ants (closed symbols) were re-introduced in their group where they could be allo-groomed (circles), re-introduced in their group where they could have social contacts through mesh but no allo-grooming (triangles) or maintained in isolation where they could only self-groom (squares). After $2.5 \mathrm{~h}$, the focal ants were kept in isolation and their survival (proportion of ants alive) was monitored for 10 days. Different letters indicate treatments that differed significantly from one another.

contacts through mesh, or isolated; $\chi^{2}=0.7$, d.f. $=2.4$, $P=0.8)$.

\section{Discussion}

By cooperating, social animals can increase their resistance to environmental stressors, predators or parasites (Andersson, 1984; Hughes et al., 2002; Andras et al., 2007; Cremer et al., 2007). Allo-grooming permits the removal of infectious particles from the body of other individuals, which is likely to increase the collective resistance to parasites at a potential cost to groomers (Schmid-Hempel, 1998; Hughes et al., 2002; Cremer et al., 2007; Clutton-Brock, 2009). Because allo-grooming combines social and hygienic functions, it is important to examine when and where it is expressed, and what are its benefits and costs in terms of disease resistance.

We found that the behavioural response of $F$. selysi ant workers to the presence of $M$. anisopliae fungal spores depended on the context and dose. When a social group was jointly exposed to a high dose of spores deposited in their nest chamber, the frequency of self-grooming increased significantly. This shows that the ants were able to detect the presence of spores, which is in line with previous studies on ants and termites (Jaccoud et al., 1999; Ugelvig \& Cremer, 2007; Mburu et al., 2009; Ugelvig et al., 2010). However, and somewhat surprisingly, this joint exposure of group-mates to spores did not result in a significant increase in the frequency of allogrooming. It is possible that the presence of spores on filter paper did not result in a high degree of contami- nation of the ants' body, even if it did cause significant mortality in previous experiments (Chapuisat et al., 2007; Reber et al., 2008).

Most individuals that were re-introduced into their group after a short or long period of isolation were immediately and intensively groomed by group-mates, independent of whether or not they had been contaminated by fungal spores. The intensity of allo-grooming was increased by the use of the surfactant Tween, when compared to the water or dry treatments. This response likely results from the ability of the surfactant to wet the hydrophobic cuticle of the ant (Rostas \& Blassmann, 2009). Hence, the grooming response appears to be increased if the ants carry an unusual chemical substance adhering to their cuticle and potentially altering their odour. Such a modulation may serve both a hygienic function and the social function of re-homogenizing the colony odour (Boulay et al., 2004).

Overall, these results suggest that allo-grooming is a proactive and prophylactic response directed towards all individuals returning to the nest, independent of the presence of fungal spores but modulated by chemical alterations. Individuals returning from exterior trips are likely to carry dangerous infectious progagules that may contaminate the nest and brood. The systematic and modulated cleaning of individuals returning to the nest by other individuals might thus serve to maintain the nest hygiene. In line with this hypothesis, leaf-cutting ant workers engage in intensive self-grooming upon entering nest chambers that contain brood or crops (Morelos Juárez et al., 2010).

The allo-grooming of uncontaminated ants lasted for longer when the re-introduction occurred after a first re-introduction of a contaminated ant. This suggests that the ants further modulate their behaviour in response to the risk of infection, which they can evaluate from previous contacts with contaminated individuals. A similar result was observed in Acromyrmex echiniator, where a second exposure to Metarhizium elicited a higher level of allo-grooming (Walker \& Hughes, 2009). This also indicates that $F$. selysi workers are able to sense the presence of fungal spores or infection and may adjust their reaction accordingly at a later stage, even if they do not immediately increase the amount of allo-grooming towards contaminated individuals.

Allo-grooming was an efficient way to remove fungal spores from the body of group-mates. In experiment 3, the contaminated ants that were kept in isolation and could only self-groom had on average 5200 spores still present on their cuticle. Contaminated ants that could be allo-groomed for $10 \mathrm{~min}$, in addition to any previous selfgrooming, had about half this number of fungal spores remaining on their body. It is likely that allo-grooming permitted the removal of spores from body parts that were beyond the reach of self-grooming individuals. Re-introduced ants were also usually groomed by several individuals, which further increased the efficiency of 
spore removal. Indeed, the total duration of allo-grooming was positively correlated with the number of allogroomers and negatively correlated with the number of conidia remaining on the surface of the groomed ant. Similarly, leaf-cutting ants and Coptotermes formosanus termites maintained in groups had fewer spores remaining on their body than individuals maintained in isolation (Hughes et al., 2002; Yanagawa \& Shimizu, 2007).

The efficiency of allo-grooming did not depend on the size of the group in which the contaminated ants were re-introduced. Moreover, when re-introduced into large groups, only a small fraction of the workers (four to six on average) groomed the contaminated ant. It thus seems possible that the number of ants that are able to efficiently groom a single individual at the same time is limited by space constraints or by some self-regulating mechanism of task allocation. The efficiency of allogrooming at removing spores also did not depend on the time elapsed between contamination and re-introduction. This might be attributed to a weak adherence of this fungus, as suggested by the fact that $M$. anisopliae conidia bond less effectively to the insect cuticle than conidia from other entomopathogenic fungi (Yanagawa et al., 2008).

In our experiments, the grooming of contaminated ants did not protect them from the fungal infection. The mortality caused by the fungal parasite was very high, as nearly $60 \%$ of the contaminated ants died from a Metarhizium infection. The survival of contaminated ants was not higher when they could be allo-groomed than when they only had social contact through a mesh preventing allo-grooming or were kept in complete social isolation. This result contrasts with several experiments that had shown that being in a group increased the survival of termites or ants challenged with $M$. anisopliae (Rosengaus et al., 1998b; Hughes et al., 2002; Traniello et al., 2002). A major difference was that in our experiment, the contaminated individuals had only a relatively short contact with nestmates (up to $2.5 \mathrm{~h}$ ) and were not maintained in the group until they died. Hence, the initial dose of spores might have been too high to permit their efficient removal by nestmates in this limited period of allo-grooming. Moreover, the ants were re-introduced $24 \mathrm{~h}$ after contamination, which is likely to reduce the effect of allo-grooming on ant survival if the conidia have already germinated and adhered to the cuticle. More generally, the curative effect of being in a group might require prolonged social interactions involving grooming, feeding or transfers of antibiotics.

The fungus strain that we used was virulent, as shown by the high mortality that it caused to experimentally contaminated ants. However, despite the high dose applied, the rate of disease transmission from contaminated ants to groomers and other group-mates was very low. In the course of our experiments, more than 1500 ants were exposed to a contaminated nestmate and only one of them died and produced Metarhizium spores. Low rates of transmission of Metarhizium have already been documented in leaf-cutting ants, Cardiocondyla ants and Zootermopsis termites (Rosengaus et al., 1998b; Hughes et al., 2002; Ugelvig et al., 2010). These low rates of disease transmission suggest that social insects are able to inactivate the spores that they remove. In several other ant and termite species, workers are indeed able to neutralize fungal spores by segregating them under nest material, destroying them mechanically by a compression in an infra-buccal pocket and/or exposing them to UV sunlight or antibiotics (Febvay et al., 1984; Rosengaus et al., 1998a; Jaccoud et al., 1999; Hughes et al., 2002; Poulsen et al., 2002; Fernandez-Marin et al., 2006; Little et al., 2006; Cremer et al., 2007). Whatever the mechanism, our results suggest that the removal of Metarhizium spores by allo-grooming is a low risk activity for the groomers.

'In summary, F. selysi workers returning to their group after a period of isolation were systematically allo-groomed by nestmates, independent of the presence of Metarhizium spores on their bodies. The intensity of the response to returning ants appears to be increased in response to a substance coating the cuticle of the returning ant, or if the group has been previously exposed to a pathogen carried by a returning ant. A short period of allo-grooming permitted the removal of a large fraction of fungal spores from the bodies of heavily contaminated workers. The allo-groomers had little risk of becoming infected, but allo-grooming did not seem to protect the contaminated ants, at least under the set of conditions tested, which involved a high initial dose of spores and one late bout allogrooming. The systematic allo-grooming of returning foragers thus seems to be a constitutive prophylactic behaviour that protects the group against the entrance of infectious propagules, rather than a behaviour elicited by the presence of the parasites and aimed at protecting already heavily contaminated or infected individuals.

Overall, worker response to contaminated nestmates seems highly variable in social insects, ranging from social avoidance (Marikovski, 1964; Hart \& Ratnieks, 2001; Ballari et al., 2007; Heinze \& Walter, 2010) to intensive allo-grooming (Rosengaus et al., 1998b; Hughes \& Boomsma, 2004; Walker \& Hughes, 2009). The allogrooming response may depend on the ecological context, including the identity of the parasite as well as the social and spatial organization of the host colony. For example, allo-grooming may be targeted at individuals that are potentially at risk of bringing infectious particles into the colony (this study) or be directed towards the most sensitive group members, such as brood (Ugelvig $\delta$ Cremer, 2007). Allo-grooming may also provide protection beyond the removal of infectious propagules if the exposure of naïve individuals to nonlethal doses of a parasite increases their future immunity (Traniello et al., 2002; Ugelvig \& Cremer, 2007). 


\section{Acknowledgments}

We thank three anonymous reviewers for comments on the manuscript. This project was supported by grants 31003A-108263 and 31003A-125306 from the Swiss National Science Foundation.

\section{References}

Andersson, M. 1984. The evolution of eusociality. Annu. Rev. Ecol. Syst. 15: 165-189.

Andras, P., Lazarus, J. \& Roberts, G. 2007. Environmental adversity and uncertainty favour cooperation. BMC Evol. Biol. 7: 240 .

Ballari, S., Farji-Brener, A.G. \& Tadey, M. 2007. Waste management in the leaf-cutting ant Acromyrmex lobicornis: division of labour, aggressive behaviour, and location of external refuse dumps. J. Insect Behav. 20: 87-98.

Boulay, R., Katzav-Gozansky, T., Hefetz, A. \& Lenoir, A. 2004. Odour convergence and tolerance between nestmates through trophallaxis and grooming in the ant Camponotus fellah (Dalla Torre). Ins. Soc. 51: 55-61.

Chapuisat, M. 2010. Social evolution: sick ants face death alone. Curr. Biol. 20: R104-R105.

Chapuisat, M., Bocherens, S. \& Rosset, H. 2004. Variable queen number in ant colonies: no impact on queen turnover, inbreeding, and population genetic differentiation in the ant Formica selysi. Evolution 58: 1064-1072.

Chapuisat, M., Oppliger, A., Magliano, P. \& Christe, P. 2007. Wood ants use resin to protect themselves against pathogens. Proc. R. Soc. Lond. B 274: 2013-2017.

Clutton-Brock, T. 2009. Cooperation between non-kin in animal societies. Nature 462: 51-57.

Cremer, S., Armitage, S.A.O. \& Schmid-Hempel, P. 2007. Social immunity. Curr. Biol. 17: R693-R702.

Dunbar, R.I.M. 2010. The social role of touch in humans and primates: behavioural function and neurobiological mechanisms. Neurosci. Biobehav. Rev. 34: 260-268.

Febvay, G., Decharme, M. \& Kermarrec, A. 1984. Digestion of chitin by the labial glands of Acromyrmex octospinosus Reich (Hymenoptera, Formicidae). Can. J. Zool. 62: 229-234.

Fefferman, N.H., Traniello, J.F.A., Rosengaus, R.B. \& Calleri, D.V. 2007. Disease prevention and resistance in social insects: modeling the survival consequences of immunity, hygienic behavior, and colony organization. Behav. Ecol. Sociobiol. 61: 565-577.

Fernandez-Marin, H., Zimmerman, J.K., Rehner, S.A. \& Wcislo, W.T. 2006. Active use of the metapleural glands by ants in controlling fungal infection. Proc. R. Soc. Lond. B 273: 16891695.

Hajek, A.E. \& St. Leger, R.J. 1994. Interactions between fungal pathogens and insect hosts. Annu. Rev. Entomol. 39: 293-322.

Hamilton, W.D. 1987. Kinship, recognition, disease, and intelligence: constraints of social evolution. In: Animal Societies: Theories and Facts (Y. Ito, J.L. Brown \& J. Kikkawa, eds), pp. 81-102. Japan Sci. Soc. Press, Tokyo.

Hart, A.G. \& Ratnieks, F.L.W. 2001. Task partitioning, division of labour and nest compartmentalisation collectively isolate hazardous waste in the leafcutting ant Atta cephalotes. Behav. Ecol. Sociobiol. 49: 387-392.
Hart, A.G. \& Ratnieks, F.L.W. 2002. Waste management in the leaf-cutting ant Atta colombica. Behav. Ecol. 13: 224-231.

Heinze, J. \& Walter, B. 2010. Moribund ants leave their nests to die in social isolation. Curr. Biol. 20: 249-252.

Hughes, W.O.H. \& Boomsma, J.J. 2004. Genetic diversity and disease resistance in leaf-cutting ant societies. Evolution 58: $1251-1260$

Hughes, W.O.H., Eilenberg, J. \& Boomsma, J.J. 2002. Trade-offs in group living: transmission and disease resistance in leafcutting ants. Proc. R. Soc. Lond. B 269: 1811-1819.

Jaccoud, D.B., Hughes, W.O.H. \& Jackson, C.W. 1999. The epizootiology of a Metarhizium infection in mini-nests of the leaf-cutting ant Atta sexdens rubropilosa. Entomol. Exp. Appl. 93: 51-61.

Keller, I. \& Zettel, J. 2001. Contribution to the autoecology of Formica selysi Bondroit, 1918 (Hymenoptera, Formicidae) in a mature steppe and a newly created alluvial zone at Pfynwald (Switzerland): I. Feeding ecology. Mitt. Schweiz. Entomol. Ges. 74: 183-193.

Keller, S., Kessler, P. \& Schweizer, C. 2003. Distribution of insect pathogenic soil fungi in Switzerland with special reference to Beauveria brongniartii and Metharhizium anisopliae. Biocontrol 48: 307-319.

Lacey, L.A. (ed.) 1997. Manual of Techniques in Insect Pathology. Academic Press, San Diego.

Little, A.E.F., Murakami, T., Mueller, U.G. \& Currie, C.R. 2006. Defending against parasites: fungus-growing ants combine specialized behaviours and microbial symbionts to protect their fungus gardens. Biol. Lett. 2: 12-16.

Marikovski, P.I. 1964. On some features of behavior of the ants Formica rufa L. infected with fungous disease. Ins. Soc. 9: 173179.

Mburu, D.M., Ochola, L., Maniania, N.K., Njagi, P.G.N., Gitonga, L.M., Ndung'u, M.W. et al. 2009. Relationship between virulence and repellency of entomopathogenic isolates of Metarhizium anisopliae and Beauveria bassiana to the termite Macrotermes michaelseni. J. Insect Physiol. 55: 774-780.

Moino, A., Alves, S.B., Lopes, R.B., Oliveira, P.M., Neves, J. \& Pereira, R.M. et al. 2002. External development of the entomopathogenic fungi Beauveria bassiana and Metarhizium anisopliae in the subterranean termite Heterotemes tenuis. Sci. Agric. 59: 267-273.

Mooring, M.S., Blumstein, D.T. \& Stoner, C.J. 2004. The evolution of parasite-defence grooming in ungulates. Biol. J. Linn. Soc. 81: 17-37.

Morelos Juárez, C., Walker, T.N., Lopes, J.F.S. \& Hughes, W.O.H. 2010. Ant farmers practice proactive personal hygiene to protect their fungus crop. Curr. Biol. 20: R553-R554.

Naug, D. 2008. Structure of the social network and its influence on transmission dynamics in a honeybee colony. Behav. Ecol. Sociobiol. 62: 1719-1725.

Poulsen, M., Bot, A.N.M., Nielsen, M.G. \& Boomsma, J.J. 2002. Experimental evidence for the costs and hygienic significance of the antibiotic metapleural gland secretion in leaf-cutting ants. Behav. Ecol. Sociobiol. 52: 151-157.

R Development Core Team (2006) R: a language and environment for statistical computing. In: $R$ Foundation for Statistical Computing. Vienna, Austria. URL: http://www.R-project.org.

Reber, A., Castella, G., Christe, P. \& Chapuisat, M. 2008. Experimentally increased group diversity improves disease resistance in an ant species. Ecol. Lett. 11: 682-689. 
Rosengaus, R.B. \& Traniello, J.F.A. 1997. Pathobiology and disease transmission in dampwood termites [Zootermopsis angusticollis (Isoptera: Termopsidae)] infected with the fungus Metarhizium anisopliae (Deuteromycotina: Hypomycetes). Sociobiology 30: 185-195.

Rosengaus, R.B., Guldin, M.R. \& Traniello, J.F.A. 1998a. Inhibitory effect of termite fecal pellets on fungal spore germination. J. Chem. Ecol. 24: 1697-1706.

Rosengaus, R.B., Maxmen, A.B., Coates, L.E. \& Traniello, J.F.A. 1998b. Disease resistance: a benefit of sociality in the dampwood termite Zootermopsis angusticollis (Isoptera: Termopsidae). Behav. Ecol. Sociobiol. 44: 125-134.

Rostas, M. \& Blassmann, K. 2009. Insects had it first: surfactants as a defence against predators. Proc. R. Soc. Lond. B 276: 633-638.

Schmid-Hempel, P. 1998. Parasites in Social Insects. Princeton University Press, Princeton.

Therneu, T.M., Grambsch, P.M. \& Pankratz, V.S. 2003. Penalized survival models and frailty. J. Comput. Graph. Stat. 12: 156-175.

Traniello, J.F.A., Rosengaus, R.B. \& Savoie, K. 2002. The development of immunity in a social insect: evidence for the group facilitation of disease resistance. Proc. Natl Acad. Sci. USA 99: 6838-6842.

Ugelvig, L.V. \& Cremer, S. 2007. Social prophylaxis: group interaction promotes collective immunity in ant colonies. Curr. Biol. 17: 1967-1971.

Ugelvig, L.V., Kronauer, D.J.C., Schrempf, A., Heinze, J. \& Cremer, S. 2010. Rapid anti-pathogen response in ant societies relies on high genetic diversity. Proc. R. Soc. Lond. B 277: 28212828.

Walker, T.N. \& Hughes, W.O.H. 2009. Adaptive social immunity in leaf-cutting ants. Biol. Lett. 5: 446-448.
Wilson-Rich, N., Spivak, M. \& Fefferman, N.H. 2009. Genetic, individual, and group facilitation of disease resistance in insect societies. Annu. Rev. Entomol. 54: 405-423.

Yanagawa, A. \& Shimizu, S. 2007. Resistance of the termite, Coptotermes formosanus Shiraki to Metarhizium anisopliae due to grooming. Biocontrol 52: 75-85.

Yanagawa, A., Yokohari, F. \& Shimizu, S. 2008. Defense mechanism of the termite, Coptotermes formosanus Shiraki, to entomopathogenic fungi. J. Invertebr. Pathol. 97: 165-170.

\section{Supporting information}

Additional Supporting Information may be found in the online version of this article:

Appendix S1 Additional control experiments.

Figure S1 This figure presents an additional control experiment to complement the experiment presented in Fig. 2 of the main text.

As a service to our authors and readers, this journal provides supporting information supplied by the authors. Such materials are peer-reviewed and may be reorganized for online delivery, but are not copy-edited or typeset. Technical support issues arising from supporting information (other than missing files) should be addressed to the authors.

Received 12 May 2010; revised 23 December 2010; accepted 4 January 2011 